\title{
A REMARK ON SOLITON-POTENTIAL INTERACTIONS FOR NONLINEAR SCHRÖDINGER EQUATIONS
}

\author{
Galina Perelman
}

\begin{abstract}
We study the interaction of small amplitude solitons with a repulsive potential $V$ for the nonlinear Schrödinger equation $i \psi_{t}=-\psi_{x x}+V(x) \psi+F\left(|\psi|^{2}\right) \psi$. We show that in the case where the nonlinearity $F(\xi)$ is $L_{2}$ critical at zero, the incoming soliton is splitted by $V$ into two outgoing waves that radiate to zero as $t \rightarrow+\infty$.
\end{abstract}

\section{Introduction}

In this note we consider the nonlinear Schrödinger equation

$$
i \psi_{t}=-\psi_{x x}+V(x) \psi+F\left(|\psi|^{2}\right) \psi, \quad(t, x) \in \mathbb{R} \times \mathbb{R},
$$

where $V$ is a "repulsive" potential, rapidly decaying as $|x| \rightarrow \infty$, and $F$ is a smooth function that satisfies $F(\xi)=-\xi^{2}+O\left(\xi^{3}\right)$, as $\xi \rightarrow 0$.

The free NLS:

$$
i \psi_{t}=-\psi_{x x}+F\left(|\psi|^{2}\right) \psi
$$

possesses solutions of special form - solitary waves (or, shortly, solitons):

$$
\begin{gathered}
e^{i \Phi} \varphi(x-b(t), E), \\
\Phi=\omega t+\gamma+\frac{1}{2} v x, b(t)=v t+c, E=\omega+\frac{v^{2}}{4}>0,
\end{gathered}
$$

where $\omega, \gamma, c, v \in \mathbb{R}$ are constants and $\varphi$ is the ground state, that is a smooth positive even exponentially decreasing solution of the equation

$$
-\varphi_{x x}+E \varphi+F\left(\varphi^{2}\right) \varphi=0 .
$$

In this note we shall be concerned with the solutions of (1) that behave as $t \rightarrow-\infty$ like a soliton $e^{i \Phi} \varphi(x-b(t), E), v \neq 0$, our goal being to understand the collision between the soliton and the potential $V$ and to determine what happens as $t \rightarrow+\infty$. We show that in the case of small amplitude solitons $(E \ll 1$ depending on $v$ ) the interaction with the potential leads to the splitting of the incoming soliton into two outgoing parts, that for large positive $t$ propagate independently according to free NLS flow (2) and radiate to zero as $t \rightarrow+\infty$. The splitting of the incoming soliton is completely controlled by the linear flow $e^{-i t L}, L=-\partial_{x}^{2}+V$ : in the interaction region the effects of the nonlinearity $F$ can be neglected, a small amplitude soliton behaves as a slowly modulated plane wave $e^{-i v^{2} t / 4+i v x / 2}$ and splits into a reflected and a transmitted parts accordingly to the linear scattering theory. For the first time

Received by the editors July 18, 2008. 
this phenomenon was observed by J.Holmer, J.Marzuola, M.Zworski [3], [4] in the context of the cubic NLS with an external delta potential:

$$
i \psi_{t}=-\psi_{x x}+q \delta(x) \psi-|\psi|^{2} \psi .
$$

The radiation of the solution to zero in the post interaction region $(t \rightarrow+\infty)$ is due to the $L_{2}$ critical nature of the nonlinearity $F$.

The structure of this paper is briefly as follows. It consists of two sections. In the first section we introduce some preliminary objects and state the main results. The second one contains the complete proofs of the indicated results.

\section{Background and statement of the results}

1.1. Assumptions and preliminary facts. Consider the nonlinear Schrödinger equation

$$
\psi_{t}=-\psi_{x x}+V(x) \psi+F\left(|\psi|^{2}\right) \psi, \quad(t, x) \in \mathbb{R} \times \mathbb{R}
$$

We assume the following.

Hypothesis H1. $V(x)$ is a real smooth exponentially decaying function:

$\left|\left(\partial^{l} V\right)(x)\right| \leq C e^{-\gamma|x|}, l=0,1, \gamma>0$, and such that the operator $L=-\partial_{x}^{2}+V$ has no eigenvalues.

Hypothesis H2. $F$ is a $C^{\infty}$ function, that satisfies: $F(\xi)=-\xi^{2}+O\left(\xi^{3}\right)$, as $\xi \rightarrow 0$.

Under assumption (H2) equation (2), for $E$ sufficiently small, has a unique positive even smooth exponentially decreasing solution $\varphi(x, E): \varphi(x, E) \sim C(E) e^{-\sqrt{E}|x|}$, as $|x| \rightarrow \infty$. Moreover, as $E \rightarrow 0, \varphi(x, E)$ admits an asymptotic expansion of the following form:

$$
\begin{gathered}
\varphi(x, E)=\varepsilon^{1 / 2} \hat{\varphi}(\varepsilon x, \varepsilon), \quad \hat{\varphi}(y, \varepsilon)=\sum_{k=0} \varepsilon^{k} \varphi_{k}(y), \quad \varepsilon=\sqrt{E}, \\
\varphi_{0}(y)=\frac{3^{1 / 4}}{\operatorname{ch}^{1 / 2} 2 y}, \quad\left|\varphi_{k}(y)\right| \leq C_{k} e^{-|y|} .
\end{gathered}
$$

The asymptotic expansion (1.2) holds in the sense:

$$
\left|\hat{\varphi}(y, \varepsilon)-\sum_{k=0}^{N} \varepsilon^{k} \varphi_{k}(y)\right| \leq C_{N} \varepsilon^{N+1} e^{-|y|},
$$

and can be differentiated any number of times with respect to $y$.

Next we recall some standard estimates of the linear evolution $e^{-i t L}$ that will be used later. $e^{-i t L}$ is an unitary group in $L_{2}$ and since $V$ is bounded, one has trivially

$$
\left\|e^{-i t L} f\right\|_{H^{k}(\mathbb{R})} \leq C\|f\|_{H^{k}(\mathbb{R})}, \quad k=0,1 .
$$

Furthermore, under assumption (H1), the following Strichartz estimates hold.

Proposition 1.1. Under assumption (H1), one has for $k=0,1$ :

$$
\begin{gathered}
\left\|e^{-i t L} f\right\|_{L_{t}^{r}\left(W_{x}^{k, p}\right)} \leq C\|f\|_{H^{k}}, \\
\left\|\int_{0}^{t} d s e^{-i(t-s) L} F(s)\right\|_{L_{t}^{r}\left(W_{x}^{k, p}\right)} \leq C\|F\|_{L_{t}^{a^{\prime}}\left(W_{x}^{k, b^{\prime}}\right)},
\end{gathered}
$$


provided $(r, p),(a, b)$ are admissible, i.e. $4<r \leq \infty$ and $\frac{2}{r}+\frac{1}{p}=\frac{1}{2}$ and the same for $(a, b)$.

By way of explanation we remark that these Strichartz inequalities can be deduced in a standard way from the following dispersive estimates:

$$
\left\|e^{-i t L} f\right\|_{W^{k, p}} \leq|t|^{-\frac{1}{2}+\frac{1}{p}}\|f\|_{W^{k, p^{\prime}}}, \quad 2 \leq p \leq \infty, \quad k=0,1,
$$

see [2], [5], [6] for the proofs.

Next we introduce some notions related to the scattering problem for the operator $L$. Let $f(x, k), g(x, k)$ be Jost solutions corresponding to $L$, i.e solutions of the equation $\left(L-k^{2}\right) u=0, k \in \mathbb{R}$, with the following asymptotic behavior as $x \rightarrow \pm \infty$

$$
\begin{aligned}
& f(x, k)=e^{i k x}\left(1+O\left(e^{-\gamma x}\right)\right), \quad x \rightarrow+\infty, \\
& g(x, k)=e^{-i k x}\left(1+O\left(e^{\gamma x}\right)\right), \quad x \rightarrow-\infty .
\end{aligned}
$$

The solutions $f, g$ are smooth function of $x$ and $k$ and the asymptotic representations (1.3), (1.4) can be differentiated with respect to both variables.

Here and below we use $\gamma$ as a general notation for the positive constants that may change from line to line.

For $k \neq 0, k \in \mathbb{R}, f(x, k), f(x,-k)=\overline{f(x, k)}$ and $g(x, k), g(x,-k)=\overline{g(x, k)}$ form two basises in the space of the solutions that are related by the transition matrix $T(k)$ :

$$
\left(\begin{array}{c}
f(k) \\
f(-k)
\end{array}\right)=T(k)\left(\begin{array}{c}
g(-k) \\
g(k)
\end{array}\right), \quad T(k)=\left(\begin{array}{cc}
\frac{a(k)}{b(k)} & \frac{b(k)}{a(k)}
\end{array}\right), \operatorname{det} T(k)=1 .
$$

We denote by $r(k)$ and $s(k)$ the reflection and transmission coefficient:

$$
r(k)=\frac{b(k)}{a(k)}, \quad s(k)=\frac{1}{a(k)}, \quad|r(k)|^{2}+|s(k)|^{2}=1 .
$$

Throughout this paper we shall suppose that $r \not \equiv 0$.

\subsection{Main results.}

Theorem 1.1. Let $v \neq 0$. Then for $\varepsilon$ sufficiently small (depending on $v$ ) equation (1.1) has a unique solution $\psi(t)$ that satisfies as $t \rightarrow-\infty$

$$
\left\|\psi(t)-w_{v, \varepsilon}(t)\right\|_{H^{1}}=O\left(e^{\gamma \varepsilon t}\right), w_{v, \varepsilon}(x, t)=e^{i \omega t+i v x / 2} \varphi\left(x-v t, \varepsilon^{2}\right), \omega=-\frac{v^{2}}{4}+\varepsilon^{2} .
$$

If $r(v / 2) \neq 0$ then $\psi$ is global $\left(\psi(t) \in C\left(\mathbb{R}, H^{1}\right)\right)$ and is purely dispersive as $t \rightarrow+\infty$ : there exists $\psi_{+} \in H^{1}$ such that

$$
\left\|\psi(t)-e^{-i t L_{0}} \psi_{+}\right\|_{H^{1}} \rightarrow 0, \quad t \rightarrow+\infty .
$$

Here $L_{0}=-\partial_{x}^{2}$.

A more detailed description of the solutions in the post-interaction region is given by the following theorem. 
Theorem 1.2. Let $v \neq 0$ and such that $r(v / 2) \neq 0$. Then for $t \geq T, T=\varepsilon^{-3 / 2}$ the solution $\psi(t)$ of (1.1) constructed in Theorem 1.1 admits the following representation:

$$
\begin{gathered}
\psi(x, t)=\psi^{r}(x, t)+\psi^{l}(x, t)+\eta(x, t), \\
\psi^{r}(x, t)=e^{-i v^{2} t / 4+i v x / 2} \varepsilon^{1 / 2} z^{+}\left(\varepsilon(x-v t), \varepsilon^{2} t\right), \\
\psi^{l}(x, t)=e^{-i v^{2} t / 4-i v x / 2} \varepsilon^{1 / 2} z^{-}\left(\varepsilon(x+v t), \varepsilon^{2} t\right),
\end{gathered}
$$

Here $z^{ \pm}(y, \tau)$ are the solutions of the $L^{2}$ critical NLS:

$$
\begin{gathered}
i z_{\tau}^{ \pm}=-z_{y y}^{ \pm}-\left|z^{ \pm}\right|^{4} z^{ \pm}, \\
\left.z^{ \pm}\right|_{\tau=0}=\alpha_{ \pm} \varphi_{0}(y), \\
\alpha_{+}=s(v / 2), \quad \alpha_{-}=r(v / 2),
\end{gathered}
$$

and the remainder $\eta$ satisfies

$$
\|\eta(t)\|_{L^{\infty}\left([T, \infty), H^{1}\right)}+\|\eta\|_{L^{6}\left([T, \infty), W^{1,6}\right)} \leq C \varepsilon^{1 / 2} .
$$

Remarks. 1. The powers $\varepsilon^{-3 / 2}$ in the definition of $T$ and $\varepsilon^{1 / 2}$ in the estimate of $\eta$ are not optimal. One can replace them by $\varepsilon^{-1-\delta}$ and $\varepsilon^{1-\delta}$ respectively, with any $\delta>0$.

2. Since $\left|\alpha^{ \pm}\right|<1, \psi^{r}, \psi^{l}$ exist globally and scatter as $t \rightarrow \infty$.

\section{Proofs}

In this section we prove theorems 1.1, 1.2. Since free NLS (2) is invariant under the transformation $x \rightarrow-x$ it is sufficient to consider the case $v>0$.

We split the analysis into two parts by considering separately the time interval $(-\infty, T], T=\varepsilon^{-3 / 2}$, where the interaction takes place, and the post-interaction region $[T,+\infty)$ where the effects of the potential become negligible.

2.1. Cauchy problem with initial conditions at $-\infty$. We are interested in the solution of (1.1) with asymptotic behavior (1.5). In this subsection we prove the existence of such a solution and analyse its behavior for times $t \leq T$.

Proposition 2.1. There exist a unique solution $\psi(t)$ of (1.1) such that

$$
\left\|\psi(t)-w_{v, \varepsilon}(t)\right\|_{H^{1}}=O\left(e^{\gamma \varepsilon t}\right), \quad t \rightarrow-\infty .
$$

Moreover,

(i) $\psi(t) \in C\left((-\infty, T], H^{1}\right)$ and admits the estimates

$$
\left\|\psi(t)-w_{v, \varepsilon}(t)\right\|_{H^{1}} \leq C \frac{e^{\gamma \varepsilon t}}{1+e^{\gamma \varepsilon t}}, \quad\|\psi(t)\|_{\infty} \leq C \varepsilon^{1 / 2}, \quad t \leq T
$$

(ii) at $t=T, \psi$ has the following form

$$
\psi(x, T)=e^{i \omega T}\left(\psi_{0}(x)+h_{0}(x)\right),
$$

$$
\psi_{0}(x)=\alpha_{-} \varepsilon^{1 / 2} e^{-i v x / 2} \varphi_{0}\left(\varepsilon\left(x+X_{0}\right)\right)+\alpha_{+} \varepsilon^{1 / 2} e^{i v x / 2} \varphi_{0}\left(\varepsilon\left(x-X_{0}\right)\right), X_{0}=v T,
$$

where the remainder $h_{0}$ satisfies

$$
\left\|h_{0}\right\|_{H^{1}} \leq C \varepsilon^{1 / 2} .
$$


Proof. We start by constructing a suitable approximate solution $W(t)$ of (1.1), (1.5). $W(t)$ is obtained as a perturbation of the profile $W^{0}(t)$ defined as follows.

$$
\begin{aligned}
W^{0}(x, t)=e^{i \omega t} \varepsilon^{1 / 2}( & \varphi\left(x-v t, \varepsilon^{2}\right) \tilde{g}(x,-v / 2)+r(v / 2) \varphi\left(x+v t, \varepsilon^{2}\right) \tilde{g}(x, v / 2) \\
& \left.+s(v / 2) \varphi\left(x-v t, \varepsilon^{2}\right) \tilde{f}(x, v / 2)\right)
\end{aligned}
$$

Here $\tilde{f}(x, k)=\theta_{+}(x) f(x, k), \tilde{g}(x, k)=\theta_{-}(x) g(x, k), \theta_{ \pm}$being the cut off function that satisfy: $\theta_{ \pm} \in C^{\infty}(\mathbb{R}), \theta_{+}+\theta_{-}=1, \theta_{ \pm}(x)=1, \quad \pm x \geq 1$.

It is easy to check that $W^{0}(t)$ has the following properties. First, $W^{0}$ satisfies estimates (2.1), (2.2), (2.3) stated in proposition 2.1. More precisely,

$$
\begin{gathered}
\left\|W^{0}(t)-w_{v, \varepsilon}(t)\right\|_{H^{1}} \leq C \frac{e^{\gamma \varepsilon t}}{1+e^{\gamma \varepsilon t}},\left\|\partial_{x}^{l} W^{0}(t)\right\|_{\infty} \leq C \varepsilon^{1 / 2}, l=0,1, t \in \mathbb{R}, \\
\left\|W^{0}(x, T)-e^{i \omega T} \psi_{0}\right\|_{H^{1}} \leq C \varepsilon .
\end{gathered}
$$

Second, $W^{0}$ solves equation (1.1) up to the error term

$$
R_{0} \equiv-i W_{t}^{0}+L W_{0}+F\left(\left|W^{0}\right|^{2}\right) W_{0}
$$

of the following structure:

$$
\begin{gathered}
R_{0}=R_{0}^{\prime}+R_{0}^{\prime \prime}, \quad R_{0}^{\prime}=e^{i \omega t} \varepsilon^{3 / 2} \varphi_{0}^{\prime}(\varepsilon v t) e(x), \\
\left\|R_{0}^{\prime \prime}(t)\right\|_{H^{1}} \leq C \varepsilon^{2} \frac{e^{\gamma \varepsilon t}}{1+e^{\gamma \varepsilon t}}, \quad t \in \mathbb{R} .
\end{gathered}
$$

Here $e$ is an exponentially decaying function of $x$ only. It is possible to give an explicit expression for it but it is useless for our purposes.

We now construct $W$ by adding to $W^{0}$ a correction $W^{1}$ that allows to get rid of $R_{0}^{\prime}$ and thus to improve the approximation. Define $W^{1}$ as follows.

$$
W^{1}(x, t)=e^{i \omega t} \varepsilon^{3 / 2}\left(\varphi_{0}^{\prime}(\varepsilon(x+v t)) \theta_{-}(x)-\varphi_{0}^{\prime}(\varepsilon(x-v t)) \theta_{+}(x)\right) H(x),
$$

where $H=-\left(L-v^{2} / 4-i 0\right)^{-1} e . H$ is in $C^{\infty}(\mathbb{R})$ and has the following asymptotic behavior at infinity:

$$
H(x)=C_{ \pm} e^{ \pm i v x / 2}\left(1+O\left(e^{\mp \gamma x}\right)\right), \quad x \rightarrow \pm \infty .
$$

A similar asymptotic representations holds for the derivatives $\partial_{x}^{k} H, k=0,1$. This implies, in particular, that

$$
\left\|W^{1}(t)\right\|_{H^{1}} \leq C \varepsilon \frac{e^{\gamma \varepsilon t}}{1+e^{\gamma \varepsilon t}},\left\|\partial_{x}^{l} W^{0}(t)\right\|_{\infty} \leq C \varepsilon^{3 / 2}, l=0,1, t \in \mathbb{R} .
$$

Set

$$
W=W^{0}+W^{1}
$$

It is not difficult to check that $W$ solves (1.1) up to the error $R \equiv-i W_{t}+L W+$ $F\left(|W|^{2}\right) W$ that satisfies

$$
\|R(t)\|_{H^{1}} \leq C \varepsilon^{2} \frac{e^{\gamma \varepsilon t}}{1+e^{\gamma \varepsilon t}}, \quad t \in \mathbb{R} .
$$

We are in position now to prove the existence of exact solution which for $t \in$ $(-\infty, T]$ is close to $W(t)$. We do it by applying a standard fixed point argument. 
More precisely, we seek a solution $\psi(t)$ of (1.1) of the form $\psi(t)=W(t)+\chi(t)$, which leads to the equation

$$
\begin{gathered}
i \chi_{t}=L \chi+N(\chi)+R \\
N(\chi)=F\left(|W+\chi|^{2}\right)(W+\chi)-F\left(|W|^{2}\right) W .
\end{gathered}
$$

For $\chi$ satisfying $\|\chi\|_{H^{1}} \leq C$, one has

$$
\begin{gathered}
\|N(\chi)\|_{H^{1}} \leq C\left(\varepsilon^{2}\|\chi\|_{H^{1}}+\|\chi\|_{H^{1}}^{5}\right), \\
\left\|N\left(\chi_{1}\right)-N\left(\chi_{2}\right)\right\|_{H^{1}} \leq C\left\|\chi_{1}-\chi_{2}\right\|_{H^{1}}\left(\varepsilon^{2}+\left\|\chi_{1}\right\|_{H^{1}}^{4}+\left\|\chi_{2}\right\|_{H^{1}}^{4}\right) .
\end{gathered}
$$

We rewrite (2.8) as an integral equation

$$
\chi(t)=(\mathbb{d} \chi)(t) \equiv-i \int_{-\infty}^{t} d s e^{-i(t-s) L}(N(\chi(s))+R(s)) .
$$

and view $\mathbb{J}$ is a mapping in the space $\left.C\left((-\infty, T], H^{1}\right)\right)$ equipped with the norm

$$
\||\chi|\|=\sup _{\tau \leq T}\left(1+e^{-\gamma \varepsilon \tau}\right)\|\chi(\tau)\|_{H^{1}} .
$$

It follows from $(2.7),(2.9),(2.10)$ that

$$
\begin{gathered}
\||\mathbb{J} \chi|\| \leq K\left(\varepsilon^{1 / 2}+\varepsilon^{1 / 2}\||\chi|\|+\varepsilon^{-3 / 2}\||\chi|\|^{5}\right), \\
\left\|\left|\mathbb{J}\left(\chi_{1}\right)-\mathbb{J}\left(\chi_{2}\right)\right|\right\| \leq K_{1}\left\|\left|\chi_{1}-\chi_{2}\right|\right\|\left(\varepsilon^{1 / 2}+\varepsilon^{-3 / 2}\left(\left\|\left|\chi_{1}\right|\right\|^{4}+\left\|\left|\chi_{2}\right|\right\|^{4}\right)\right),
\end{gathered}
$$

with some constants $K, K_{1}$. This means that $\mathbb{J}$ is a contraction of the ball $\||\chi|\| \leq$ $2 K \varepsilon^{1 / 2}$ into itself, provided $\varepsilon$ is sufficiently small. Consequently, it has a unique fixed point $\chi$ that satisfies

$$
\|\chi(t)\|_{H^{1}} \leq C \varepsilon^{1 / 2} \frac{e^{\gamma \varepsilon t}}{1+e^{\gamma \varepsilon t}}, \quad t \leq T .
$$

Combining (2.4), (2.5), (2.6), (2.11) one obtains estimates (2.1), (2.3) of proposition 2.1 .

To prove the uniqueness, one can use a similar fixed point argument, applying it to the integral equation

$$
\begin{gathered}
\tilde{\chi}=-i \int_{-\infty}^{t} d s e^{-i(t-s) L} \tilde{N}(\tilde{\chi}(s)), \\
\tilde{\chi}=\psi-w_{v, \varepsilon}, \quad \tilde{N}(\tilde{\chi})=F\left(\left|w_{v, \varepsilon}+\tilde{\chi}\right|^{2}\right)\left(w_{v, \varepsilon}+\tilde{\chi}\right)-F\left(\left|w_{v, \varepsilon}\right|^{2}\right) w_{v, \varepsilon} .
\end{gathered}
$$

This completes the proof of proposition 2.1. 
2.2. Post- interaction region $t \geq T$. By proposition 1.1 we are left with the following Cauchy problem:

$$
\begin{gathered}
i \psi_{t}=L \psi+F(|\psi|) \psi, \quad t \geq 0, \\
\left.\psi\right|_{t=0}=\psi_{0}+h_{0} \in H^{1}, \\
\psi_{0}=\alpha_{-} e^{-i v x / 2} \varepsilon^{1 / 2} \varphi_{0}\left(\varepsilon\left(x+X_{0}\right)\right)+\alpha_{+} e^{i v x / 2} \varepsilon^{1 / 2} \varphi_{0}\left(\varepsilon\left(x-X_{0}\right)\right) \\
\left\|h_{0}\right\|_{H^{1}} \leq C \varepsilon^{1 / 2}, \quad X_{0}=v \varepsilon^{-3 / 2} .
\end{gathered}
$$

The initial value problem for equation (2.12) is known to be locally well posed in $H^{1}$ (see, for example, [1]). Thus, (2.12), (2.13) has a unique solution $\psi(t) \in C\left(\left[0, T^{*}\right), H^{1}\right)$ with some $T^{*}>0$ and either $T^{*}=+\infty$ (the solution is global) or $T^{*}<\infty$ and then $\|\psi(t)\|_{H^{1}} \rightarrow \infty$ as $t \rightarrow T$. Furthermore, the Strichartz estimates of proposition 1.1 (with $r=a=6$ ) imply in a standard way that $\psi \in L^{6}\left(\left[0, t_{1}\right], W^{1,6}\right)$ for any $t_{1}<T^{*}$ and if $\psi(t)$ is global and uniformly bounded in $H^{1}$ and has in addition a finite global Strichartz norm $\|\psi\|_{L^{6}\left(\mathbb{R}_{+}, W^{1,6}\right)}$ then it scatters as $t \rightarrow+\infty$ : there exists a scattering state $\psi_{\infty} \in H^{1}$ such that

$$
\left\|\psi(t)-e^{-i L t} \psi_{\infty}\right\|_{H^{1}} \rightarrow 0, \quad t \rightarrow+\infty .
$$

Observe also that using the linear scattering theory one can rewrite (2.14) in the form

$$
\left\|\psi(t)-e^{-i L_{0} t} \psi_{+}\right\|_{H^{1}} \rightarrow 0, \quad t \rightarrow+\infty,
$$

where $\psi_{+} \in H^{1}$ is related to $\psi_{\infty}$ by means of the wave operator corresponding to the pair $L_{0}, L$. In light of this discussion the proof of theorem 1.1 is reduced to showing that for all $t_{1}<T^{*}$ one has a uniform bound

$$
\|\psi(t)\|_{L^{\infty}\left(\left[0, t_{1}\right], H^{1}\right)}+\|\psi\|_{L^{6}\left(\left[0, t_{1}\right], W^{1,6}\right)} \leq C .
$$

The rest of the subsection is devoted to the proof of this estimate. We start by constructing a suitable approximate solution of (2.12), (2.13) which is built up in terms of the following Cauchy problem for the $L_{2}$ critical NLS:

$$
\begin{gathered}
i z_{t}=-z_{x x}-|z|^{4} z, \\
\left.z\right|_{t=0}=\alpha \varphi_{0}, \quad \alpha \in \mathbb{C},|\alpha|<1 .
\end{gathered}
$$

Applying the pseudo-conformal transformation, one can write $z$ as

$$
z(x, t)=\frac{e^{\frac{i x^{2}}{4(t+1)}}}{(t+1)^{1 / 2}} u^{\alpha}\left(\frac{x}{t+1},-\frac{1}{t+1}\right),
$$

where $u^{\alpha}$ solves

$$
\begin{gathered}
i u_{t}^{\alpha}=-u_{x x}^{\alpha}-\left|u^{\alpha}\right|^{4} u^{\alpha}, \\
\left.u^{\alpha}\right|_{t=-1}=\alpha e^{-i x^{2} / 4} \varphi_{0}(x) .
\end{gathered}
$$

Since $|\alpha|<1, u^{\alpha}(t)$ exists globally and is in $C(\mathbb{R}, \mathbb{S})$.

Next we introduce a modified profile $u^{\alpha, \varepsilon}$ which is defined as a solution of the Cauchy problem:

$$
\begin{gathered}
i u_{t}^{\alpha, \varepsilon}=-u_{x x}^{\alpha, \varepsilon}-\left|u^{\alpha, \varepsilon}\right|^{4} u^{\alpha, \varepsilon}, \\
\left.u^{\alpha, \varepsilon}\right|_{t=0}=\theta\left(\frac{|x| \varepsilon}{v}\right) u^{\alpha}(x, 0) .
\end{gathered}
$$

Here $\theta \in C_{0}^{\infty}(\mathbb{R})$ is a cut off function: $0 \leq \theta(\xi) \leq 1, \theta(\xi)=\left\{\begin{array}{ll}1, & |\xi| \leq \frac{1}{4} \\ 0, & |\xi| \geq \frac{1}{2}\end{array}\right.$. 
Clearly, $u^{\alpha, \varepsilon} \in C(\mathbb{R}, \mathbb{S})$, uniformly with respect to $\varepsilon$ sufficiently small, and satisfies for any $N$

$$
\left\|u^{\alpha, \varepsilon}(\tau)-u^{\alpha}(\tau)\right\|_{\Sigma_{1}} \leq C_{N} \varepsilon^{N}, \quad-1 \leq \tau \leq 0 .
$$

Here $\|f\|_{\Sigma_{1}}=\|x f\|_{H^{1}}+\|f\|_{H^{2}}$.

The approximate solution $\Psi(t)$ of $(2.12),(2.13)$ can be now defined as follows.

$$
\begin{gathered}
\Psi(t)=\psi^{-}(t)+\psi^{+}(t), \\
\psi^{ \pm}(t)=e^{-i v^{2} t / 4 \pm i v x / 2} \varepsilon^{1 / 2} \tilde{\psi}^{ \pm}\left(\varepsilon\left(x \mp v t \mp X_{0}\right), \varepsilon^{2} t\right), \\
\tilde{\psi}^{ \pm}(x, t)=\frac{e^{\frac{i x^{2}}{4(t+1)}}}{(t+1)^{1 / 2}} u^{ \pm}\left(\frac{x}{t+1},-\frac{1}{t+1}\right), \\
u^{ \pm}=u^{\alpha_{ \pm}, \varepsilon} .
\end{gathered}
$$

The basic properties of the profile $\Psi(x, t)$ are collected in the following proposition.

Proposition 2.2. (i) $\Psi$ satisfies the initial conditions (2.13) in the sense

$$
\|\Psi(0)-\psi(0)\|_{H^{1}} \leq C \varepsilon^{1 / 2},
$$

(ii) $\Psi(t)$ admits the estimate

$$
\|\Psi(t)\|_{W^{k, p}} \leq C\left(\frac{\varepsilon}{1+\varepsilon^{2} t}\right)^{\frac{1}{2}-\frac{1}{p}}, \quad t \geq 0, \quad k=0,1, \quad 2 \leq p \leq \infty,
$$

(iii) the error $r \equiv-i \Psi_{t}+L \Psi+F\left(|\Psi|^{2}\right) \Psi$ satisfies

$$
\|r\|_{L^{6 / 5}\left(\mathbb{R}_{+}, W^{1,6 / 5}\right)} \leq C \varepsilon .
$$

Proof.

Part (i) follows directly from (2.16).

Estimate (ii) is a consequence of the bound

$$
\left\|<z>^{m} u^{\alpha, \varepsilon}(\tau)\right\|_{H^{l}} \leq C_{l, m},
$$

that holds for any $l, m$, uniformly with respect to $\tau \in[0,1]$ and $\varepsilon$ sufficiently small.

To estimate the error $r$ we write it as a sum $r=r_{0}+r_{1}+r_{2}$, where

$$
\begin{gathered}
r_{0}=V(x) \Psi, \quad r_{1}=-\left|\psi^{-}+\psi^{+}\right|^{4}\left(\psi^{-}+\psi^{+}\right)+\left|\psi^{-}\right|{ }^{4} \psi^{-}+\left|\psi^{+}\right|^{4} \psi^{+}, \\
r_{2}=\tilde{F}\left(|\Psi|^{2}\right) \Psi, \quad \tilde{F}(\xi)=F(\xi)+\xi^{2} .
\end{gathered}
$$

We first consider $r_{0}$. For any $l, m$, one has

$$
<z>^{m}\left|\partial_{z}^{l}\left(u^{\alpha, \varepsilon}(z, \tau)-u^{\alpha, \varepsilon}\left(z, \tau_{1}\right)\right)\right| \leq C_{l, m}\left|\tau-\tau_{1}\right|, \quad z \in \mathbb{R},
$$

uniformly with respect to $\tau, \tau_{1} \in[0,1]$ and $\varepsilon$ sufficiently small. Taking into account the support properties of $u^{ \pm}(0)\left(\operatorname{supp} u^{ \pm}(0) \subset\left\{|z| \leq \frac{v}{2 \varepsilon}\right\}\right)$, one can easily deduce from this inequality that for any $N$,

$$
\left|\partial_{x}^{l}(V(x) \Psi(x, t))\right| \leq C_{N} e^{-\gamma|x|} \frac{\varepsilon^{N}}{\left(1+\varepsilon^{2} t\right)^{3 / 2}}, \quad l=0,1 .
$$

In particular, this means that

$$
\left\|r_{0}\right\|_{L^{6 / 5}\left(\mathbb{R}_{+}, W^{1,6 / 5}\right)} \leq C_{N} \varepsilon^{N} .
$$

Consider $r_{1}$ :

$$
\left|r_{1}\right| \leq C\left|\psi^{-}\right|\left|\psi^{+}\right|\left(\left|\psi^{-}\right|^{3}+\left|\psi^{+}\right|^{3}\right),
$$


which implies

$$
\left\|r_{1}(t)\right\|_{6 / 5} \leq C\left(\frac{\varepsilon}{1+\varepsilon^{2} t}\right)^{2 / 3}\left\|\psi^{-} \psi^{+}\right\|_{\infty}
$$

Similarly,

$$
\left\|\partial_{x} r_{1}\right\|_{6 / 5} \leq C\left(\frac{\varepsilon}{1+\varepsilon^{2} t}\right)^{2 / 3}\left(\left\|\psi^{+} \partial_{x} \psi^{-}\right\|_{\infty}+\left\|\psi^{+} \partial_{x} \psi^{-}\right\|_{\infty}\right) .
$$

The expressions $\left|\psi^{ \pm} \partial_{x}^{l} \psi^{\mp}\right|, l=0,1$ can be estimated as follows:

$$
\left\|\psi^{ \pm} \partial_{x}^{l} \psi^{\mp}\right\|_{\infty} \leq C_{N} \frac{\varepsilon^{N}}{1+\varepsilon^{2} t} .
$$

Here we have once more used (2.20).

Putting the last three inequalities together one gets

$$
\left\|r_{1}\right\|_{L^{6 / 5}\left(\mathbb{R}_{+}, W^{1,6 / 5}\right)} \leq C_{N} \varepsilon^{N} .
$$

Finally, it follows from (2.18) that

$$
\left\|r_{2}\right\|_{L^{6 / 5}\left(\mathbb{R}_{+}, W^{1,6 / 5}\right)} \leq C \varepsilon
$$

This concludes the proof of proposition 2.2 .

We are now in position to prove the following uniform bounds for the difference $h(t)=\psi(t)-\Psi(t)$.

Proposition 2.3. For all $t_{1}<T^{*}$ one has

$$
\|h(t)\|_{L^{\infty}\left(\left[0, t_{1}\right], H^{1}\right)}+\|h\|_{L^{6}\left(\left[0, t_{1}\right], W^{1,6}\right)} \leq C \varepsilon^{1 / 2} .
$$

Proof. The proof is based on the standard perturbation argument. We first estimate $\|h\|_{L^{6}\left(\left[0, t_{1}\right], W^{1,6}\right)} . h(t)$ solves the Cauchy problem:

$$
\begin{gathered}
i h_{t}=L h+r+N_{1}(h), \\
\left.h\right|_{t=0}=h_{1}, \quad h_{1}=\psi(0)-\Psi(0) .
\end{gathered}
$$

Here

$$
N_{1}(h)=F\left(|\Psi+h|^{2}\right)(\Psi+h)-F\left(|\Psi|^{2}\right) \Psi .
$$

For $h$ satisfying $|h| \leq C, N_{1}(h)$ can be estimated as follows

$$
\begin{gathered}
\left|N_{1}(h)\right| \leq C\left(|h||\Psi|^{4}+|h|^{5}\right), \\
\left|\partial_{x} N_{1}(h)\right| \leq C\left(\left|h_{x}\right|\left(|\Psi|^{4}+|h|^{4}\right)+|h|\left(|\Psi|+\left|\Psi_{x}\right|\right)^{4}+|h|^{5}\right) .
\end{gathered}
$$

As a consequence,

$$
\left\|N_{1}(h(t))\right\|_{W^{1,6 / 5}} \leq C\left(\|h(t)\|_{H^{1}}\left(\frac{\varepsilon}{1+\varepsilon^{2} t}\right)^{5 / 3}+\|h(t)\|_{W^{1,6}}^{5}\right),
$$

and

$$
\left\|N_{1}(h(t))\right\|_{L^{6 / 5}\left(\left[0, t_{1}\right], W^{1,6 / 5}\right)} \leq C\left(\|h(t)\|_{L^{\infty}\left(\left[0, t_{1}\right], H^{1}\right)}+\|h(t)\|_{L^{6}\left(\left[0, t_{1}\right], W^{1,6}\right)}^{5}\right),
$$

provided $\|h(t)\|_{L^{\infty}\left(\left[0, t_{1}\right], H^{1}\right)} \leq C$.

Rewritings (2.22) as the integral equation

$$
h(t)=e^{-i t L} h_{1}-i \int_{0}^{t} d s e^{-i t L}\left(r(s)+N_{1}(h(s))\right),
$$


and applying proposition 1.1, one gets from (2.17), (2.19), (2.25):

$$
\|h\|_{L^{6}\left(\left[0, t_{1}\right], W^{1,6}\right)} \leq C\left(\varepsilon^{1 / 2}+\|h(t)\|_{L^{\infty}\left(\left[0, t_{1}\right], H^{1}\right)}+\|h\|_{L^{6}\left(\left[0, t_{1}\right], W^{1,6}\right)}^{5}\right) .
$$

In order to estimate $\|h\|_{H^{1}}$ we use the identities:

$$
\begin{aligned}
\frac{d}{d t}\|h(t)\|_{2}^{2} & =2 \operatorname{Im}\left(r+N_{1}(h), h\right) \\
\frac{d}{d t}(L h(t), h(t)) & =2 \operatorname{Im}\left(r+N_{1}(h), L h\right) .
\end{aligned}
$$

By (2.18), (2.23), (2.24), they imply:

$$
\begin{aligned}
& \frac{d}{d t}\|h(t)\|_{2}^{2} \leq C\left[\left(\frac{\varepsilon}{1+\varepsilon^{2} t}\right)^{2}\|h(t)\|_{2}^{2}+\|h(t)\|_{6}\|r(t)\|_{6 / 5}+\|h(t)\|_{6}^{6}\right], \\
& \frac{d}{d t}(L h, h)(t) \leq C\left[\left(\frac{\varepsilon}{1+\varepsilon^{2} t}\right)^{2}\|h\|_{H^{1}}^{2}+\|h\|_{W^{1,6}}\|r\|_{W^{1,6 / 5}}+\|h\|_{W^{1,6}}^{6}\right] \\
& \leq C\left[\left(\frac{\varepsilon}{1+\varepsilon^{2} t}\right)^{2}((L h, h)+(h, h))+\|h\|_{W^{1,6}}\|r\|_{W^{1,6 / 5}}+\|h\|_{W^{1,6}}^{6}\right] .
\end{aligned}
$$

As a consequence:

$$
\frac{d}{d t} y(t) \leq C\left[\left(\frac{\varepsilon}{1+\varepsilon^{2} t}\right)^{2} y(t)+\|h(t)\|_{W^{1,6}}\|r(t)\|_{W^{1,6 / 5}}+\|h(t)\|_{W^{1,6}}^{6}\right],
$$

where $y(t)=(L h(t), h(t))+(h(t), h(t))$. Integrating this inequality and using (2.17), (2.18), one gets

$$
\|h\|_{L^{\infty}\left(\left[0, t_{1}\right], H^{1}\right)}^{2} \leq C\left(\varepsilon+\varepsilon\|h\|_{L^{6}\left(\left[0, t_{1}\right], W^{1,6}\right)}++\|h\|_{L^{6}\left(\left[0, t_{1}\right], W^{1,6}\right)}^{6}\right) .
$$

Combining (2.26), (2.27), one obtains the bounds announced in proposition 2.3:

$$
\|h(t)\|_{L^{\infty}\left(\left[0, t_{1}\right], H^{1}\right)}+\|h\|_{L^{6}\left(\left[0, t_{1}\right], W^{1,6}\right)} \leq C \varepsilon^{1 / 2},
$$

which together with (2.18) implies (2.15). This completes the proof of theorem 1.1. Theorem 1.2 follows directly from proposition 2.3 and (2.16), (2.21).

\section{References}

[1] T.Cazenave, Semilinear Schrödinger equations, Courant Lecture Notes in Mathematics 10, 2003.

[2] M.Goldberg, W.Schlag, Dispersive estimates for Schrödinger operators in dimensions one and three, Comm. Math. Phys. 251 (2004), no. 1, 157-178.

[3] J.Holmer, J.Marzuola, M.Zworski, Fast soliton scattering by delta impurities, Comm. Math. Phys. 274 (2007), no. 1, 187-216.

[4] J.Holmer, J.Marzuola, M.Zworski, Soliton splitting by external delta potentials, J. Nonlinear Sci. 17 (2007), no. 4, 349-367.

[5] J.Krieger, W.Schlag, Stable manifolds for all monic supercritical focusing nonlinear Schrödinger equations in one dimension, J. Amer. Math. Soc. 19 (2006), no. 4, 815-920

[6] W.Schlag, Dispersive estimates for Schrödinger operators: a survey in Mathematical aspects of nonlinear dispersive equations, 255-285, Ann. of Math. Stud., 163, 2007.

Centre de Mathématiques, Ecole Polytechnique, F-91128 Palaiseau Cedex

E-mail address: perelmanmath.polytechnique.fr 